\title{
Development and validation of an inexpensive home-made laparoscopic trainer for surgical training
}

\author{
Chin Li Tee ${ }^{a, *}$ and David Alexander Grieve ${ }^{a, b}$ \\ ${ }^{a}$ Department of Surgery, Nambour Hospital, Hospital Road, Nambour, Queensland 4560, Australia; ${ }^{b}$ School of Medicine, Griffith \\ University, Queensland QLD 4122, Australia
}

${ }^{*}$ Corresponding author at: Nambour Hospital, Hospital Road, Nambour, Queensland 4560, Australia.

Email: tee_jimmy@hotmail.com

Date accepted for publication: 16 July 2020

\section{Abstract}

Introduction: The main hindrances to laparoscopic surgical simulation are the cost and availability of laparoscopic trainers in regional/rural hospitals. The aims of this study are to describe the development of an inexpensive homemade (HM) laparoscopic trainer for the purpose of surgical training and to validate it by comparing with the Fundamentals of Laparoscopic Surgery (FLS) trainer. Materials and methods: The HM laparoscopic trainer was constructed with a Baxter cardboard box and some inexpensive materials. A high-definition C525 Logitech webcam was mounted on the inside of the box and connected to a laptop. Participants $(n=30)$ who were postgraduate year (PGY) 1-5 were prospectively randomized to perform three tasks (peg transfer, pattern cut and placement of a ligating loop) on both the HM trainer and FLS trainer. A simple paired $t$ test was performed to compare the times between the trainers. Results: The home-made trainer was constructed in $30 \mathrm{~min}$ for approximately AUD $\$ 93.70$ (laptop ownership assumed), representing a significant cost saving. Results of the validation study demonstrated no statistical difference in times to complete 1 of 3 tasks $(P<0.05)$. On a 10-point Likert scale, the HM trainer was given a median score of between 8 and 9 for performance (video quality, lighting, simplicity of setup) and 9 for training capacity. Conclusion: The home-made laparoscopic trainer can be constructed cheaply and easily. It is comparable with the FLS trainer and is a viable alternative for trainees who wish to practise basic laparoscopic skills at home.

Keywords: inexpensive; home-made; laparoscopic trainer

\section{Introduction}

Laparoscopic surgery has become established as the first-line approach for a large number of procedures in general surgery and other surgical specialties. However, laparoscopic skills are associated with a steep learning curve, because they require a different set of skills, dexterity and handeye coordination. Adding to the challenge is the work hour restriction imposed on trainees and junior doctors, resulting in less exposure and practice in laparoscopic surgery, thereby hindering mastery of laparoscopic skills.

A solution to this problem is the use of laparoscopic box trainers, which can help trainees develop their basic skills in a safe environment before applying it in real patients in the operating theatre; this practice, therefore, accelerates the learning process. ${ }^{1}$ Studies have shown that the use of box trainers leads to better acquisition of laparoscopic skills than training without these adjuncts, ${ }^{2,3}$ and even improves operating theatre performance. ${ }^{4}$ However, many such laparoscopic trainers are expensive and may not be readily accessible to surgical trainees or junior doctors, especially in rural/regional hospitals.

In this study, we aim to present a step-by-step guide for the easy construction of an inexpensive home-made (HM) laparoscopic trainer for the purpose of surgical training. To ensure the HM trainer is effective when used, we conducted a validation study comparing the HM trainer with the Fundamentals of Laparoscopic Surgery (FLS) trainer. The FLS trainer is the box trainer currently used in the FLS course, an extensively validated simulation-based educational curriculum sponsored by the Society of American Gastrointestinal and Endoscopic Surgeons and the American College of Surgeons. ${ }^{5}$

\section{Materials and methods}

The steps involved in the construction of the HM laparoscopic trainer (Fig. 1) are outlined here. The final product is 


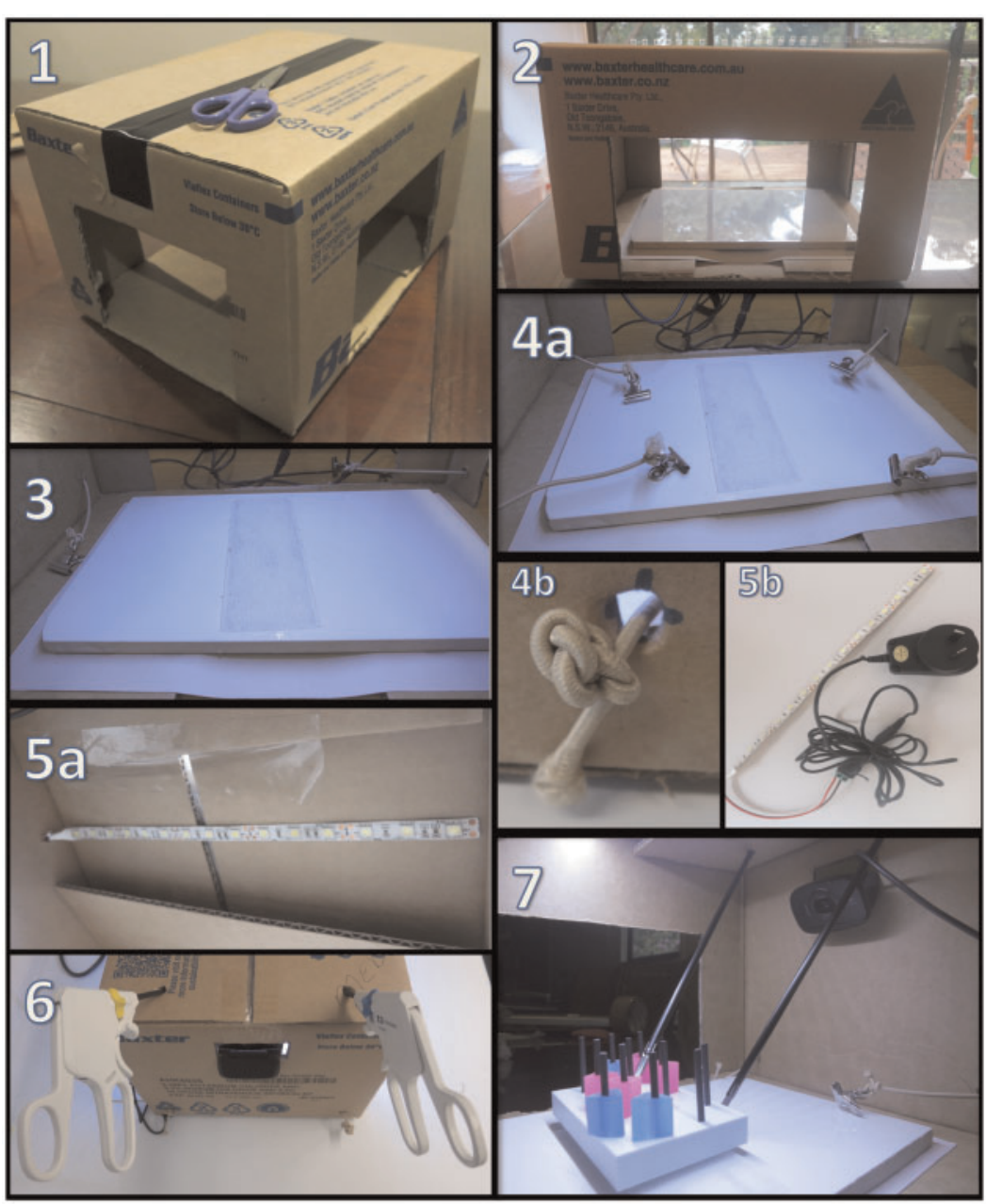

Figure 1. Steps in building a home-made laparoscopic trainer.

shown in Fig. 2. A cost breakdown of the materials used for construction is presented in Table 1 .

(1) The two lateral sides and front side of the Baxter (6) box (easily obtainable from any hospital; http://www.baxterhealthcare.com.au) are cut. The exact dimensions do not matter as long as they allow objects to be moved in and out

(2) A white tile is fixed to the base of the box using Super Glue

(3) A strip of Velcro is glued on the tile

(4) Four pieces of elastic band, each tied to a clip, are attached to four corners of the box

(5) A row of light emitting diode (LED) ribbon strip, $25 \mathrm{~cm}$ in length, is glued on the inside of the roof of the box

(6) Two holes $12 \mathrm{~cm}$ apart are made on the box to allow insertion of laparoscopic instruments (ports are not needed)

(7) A rectangular-shaped hole is cut in the user-facing side of the box to allow mounting of a high-definition C525 Logitech webcam. This hole should be placed as superior and central as possible
A prospective validation study was performed comparing the HM trainer and FLS trainer. We recruited 30 doctors from Nambour Hospital to participate in the validation study. To be eligible for the study, the participants had to be in postgraduate year (PGY) $1-5$, currently working in a surgical specialty (general surgery, urology, vascular, ear nose throat surgery, obstetrics and gynaecology). Participants $(n=30)$ were required to fill in a pre-test questionnaire before the study to establish their eligibility. Consents were obtained at the same time. The participants were asked to perform the three standardized laparoscopic FLS module tasks: peg transfer, pattern cut and insertion of an endo-loop. They were required to watch a detailed instructional video that explained and demonstrated the steps of each task before the test day. On the test day, they were randomized to begin with either the FLS trainer or the HM trainer. Each participant had to perform each task once in sequential order on the assigned trainer. After the completion of three tasks, the participants crossed over 


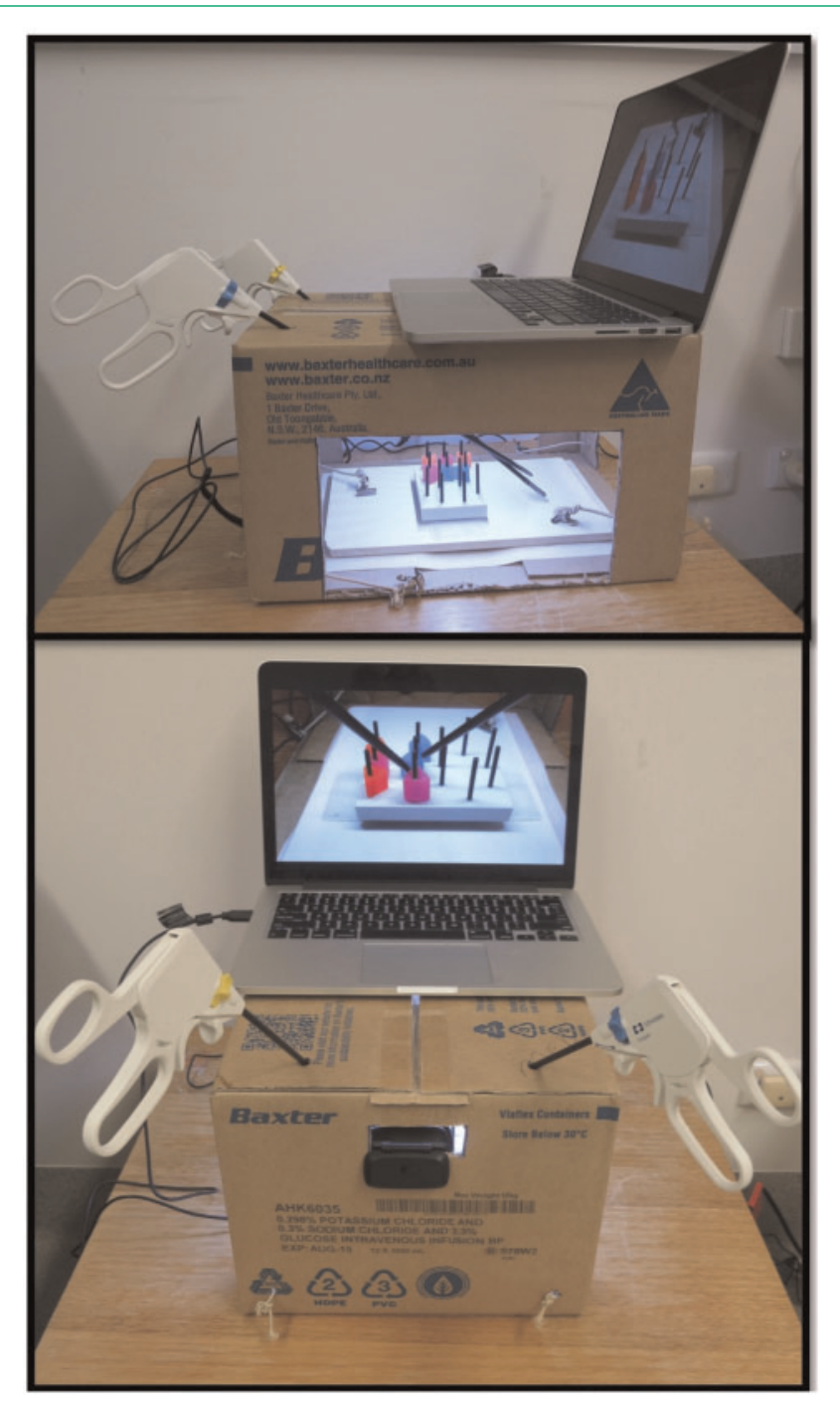

Figure 2. Home-made trainer: the final product.

to perform the same three tasks on the other trainer. Performance was scored by the time required to complete each task.At the end of the task, participants had to fill in a feedback form on the HM trainer. For post-test feedback, we asked the participants to rate the HM trainer according to performance and training capacity, with a score from 1 to 10. For performance, the participants had to rate the trainer for its video quality, lighting, simplicity of setup and overall rating. For training capacity, we asked the participants if the trainer could help develop hand-eye coordination, or develop and maintain laparoscopic skills.

Statistical analysis was performed using Stata 12 (StataCorpLP., College Station, TX, USA). The paired $t$ test was used to compare the mean times to complete each task on each trainer. $P$ values $<0.05$ were considered to be significant.
Table 1. Materials required to build home-made laparoscopic trainer

\begin{tabular}{lll}
\hline Items & Source & Cost $^{*}$ \\
\hline Logitech C525 HD webcam & $\begin{array}{c}\text { Umart online, ebay or any } \\
\text { electronic store }\end{array}$ & $\$ 56$ \\
LED ribbon light strip $25 \mathrm{~cm}$ & $\begin{array}{l}\text { Jaycar } \\
\text { Metal clips }\end{array}$ & $\$ 27.4$ \\
Elastic band $(1 \mathrm{~m})$ & Lincraft & $\$ 2.80$ \\
Super Glue & Lincraft & $\$ 1.00$ \\
White tile & Bunning's Warehouse & $\$ 1.00$ \\
Velcro & Lincraft & $\$ 2.00$ \\
Baxter box & Hospital & Free \\
Laparoscopic instruments & Covidien (donation) & Free \\
Laptop & Own & Free \\
Total & & $\$ 93.70$ \\
\hline
\end{tabular}

* Cost in AUD October 2017

Ethics approval was obtained from The Prince Charles Hospital Human Research Ethics Committee (HREC/15/ QPCH/48).

\section{Results}

Construction of the home-made laparoscopic trainer The HM trainer was constructed in 30 min using the materials shown in Table 1. The total cost was AUD\$93.70 in October 2019 (laptop ownership assumed). The laparoscopic scissors and graspers were disposable instruments accessible to any trainee. The design of the final product is simple, elegant and similar to the FLS trainer. The dimensions of our HM trainer were $35.5 \mathrm{~cm}$ (length) $\times 27.1 \mathrm{~cm}$ (width) $\times 21.3 \mathrm{~cm}$ (height) compared with the FLS trainer, which measures $48.3 \mathrm{~cm}$ (length) $\times 37.5 \mathrm{~cm}$ (width) $\times 20.3 \mathrm{~cm}$ (height). The distance between the two working ports is the same in the HM and FLS trainers at $12 \mathrm{~cm}$ apart. Thus, the amount of working space in both trainers is similar.

\section{Validation study}

A total of 30 doctors participated in the validation study. For peg transfer, the participants were significantly faster when completing the task using the FLS trainer (148.8 s; range, 89.5-214.8 s) than the HM trainer (195.8 s; range, 110.0-354.6 s) $(P=0.0001)$. The time difference was $47 \mathrm{~s}$. For pattern cut, the participants were significantly faster in completing the task using the HM trainer (176.5 s; range, 91.5-338.0 s) than the FLS trainer (202.3 s; range, 104.7-323 s) $(P=0.039)$. The time difference was $25.8 \mathrm{~s}$. There was no significant difference in the average time to complete endo- 
Table 2. Mean times to complete each task with the home-made trainer versus the FLS trainer

\begin{tabular}{|c|c|c|c|c|c|}
\hline & Number & Mean (s) & Range (s) & SEM & $\begin{array}{l}P \\
\text { value }\end{array}$ \\
\hline \multicolumn{6}{|l|}{ Peg transfer } \\
\hline HM trainer & 30 & 195.8 & $110-354.6$ & 12.79 & 0.0001 \\
\hline FLS trainer & 30 & 148.8 & $89.5-214.8$ & 9.47 & \\
\hline \multicolumn{6}{|l|}{ Pattern cut } \\
\hline HM trainer & 30 & 176.5 & $91.5-338$ & 12.24 & 0.039 \\
\hline FLS trainer & 30 & 202.3 & $104.7-323$ & 10.57 & \\
\hline \multicolumn{6}{|c|}{ Endo-loop insertion } \\
\hline HM trainer & 30 & 99.8 & $28.2-178.6$ & 6.39 & 0.791 \\
\hline FLS trainer & 30 & 101.8 & $46-167.6$ & 6.64 & \\
\hline
\end{tabular}

Bold indicates a statistically significant difference.

HM, home-made; FLS, Fundamentals of Laparoscopic Surgery; SEM, standard error of the mean.

loop insertion between the HM trainer (99.8 s; range, 28.2$178.6 \mathrm{~s}$ ) and the FLS trainer (101.8 s; range, 46-167.6 s) ( $P$ $=0.791)$ (Table 2). The time difference was $2 \mathrm{~s}$.

In general, we received good feedback on the HM trainer. For performance, the participants gave a median score of 8 for video quality and overall rating, and 9 for lighting and simplicity of setup. For training capacity, they gave a median score of 9 for hand-eye coordination, developing laparoscopic skills and maintaining laparoscopic skills. The median score for each parameter is presented in Table 3.

We also asked the participants if they would use the HM trainer if it were provided for them, and if they would consider making their own HM trainer to help with their surgical training. Twenty eight of $30(93.3 \%)$ participants stated they would use it and 26 of $30(86.7 \%)$ stated they would make their own HM trainer. Overall, the feedback was very positive and participants considered the HM trainer a creative and economical alternative, with training capacity comparable to that of the FLS trainer.

\section{Discussion}

Surgical simulation is an effective teaching tool and is often the first step in a surgical trainee's education in laparoscopic procedures. It enables trainees to learn and practise laparoscopic skills without having to rely entirely on operative opportunities, and provides a consistent environment for basic skill training. ${ }^{6}$ Such repetitive, goal-directed practice of psychomotor skills may allow some of the skills to become automated, thereby allowing trainees to shift their attention from the required manual skill to focus on the cognitive aspects of the tasks. ${ }^{7}$
Table 3. Performance and training capacity of the home-made trainer: participant feedback scores

\begin{tabular}{ll}
\hline & $\begin{array}{l}\text { Median } \\
\text { score }\end{array}$ \\
\hline Performance & \\
Video quality & 8 \\
Lighting & 9 \\
Simplicity of setup & 9 \\
Overall & 8 \\
Training capacity & \\
Hand-eye coordination & 9 \\
Developing laparoscopic skills & 9 \\
Maintaining laparoscopic skills & 9 \\
\hline
\end{tabular}

There are many commercial box trainer products available on the market. ${ }^{8,9}$ However, the cost and availability of box trainers are often the prohibitive factors in their wide adoption in training. By constructing an inexpensive HM trainer, we have addressed these two barriers. The cost comparison of the HM with the FLS trainer demonstrated a significant difference of AUD $\$ 93.70$ versus AUD \$2300 (US\$1536). The conversion rate at the time of writing was 1:1.5 (US:AUD).

Many HM trainers have been described in the literature with a wide variation in designs, sizes and capabilities. These include the use of common household materials (shoe box, plastic box, mirrors) ${ }^{1,10-13}$ or widely used consumer technology (iPhone, tablets as camera) ${ }^{14,15}$ to create low-cost training devices. The main disadvantages of some of these trainers are that some are large and cumbersome and use old materials that are no longer available nowadays, whereas others can be difficult to construct especially for 


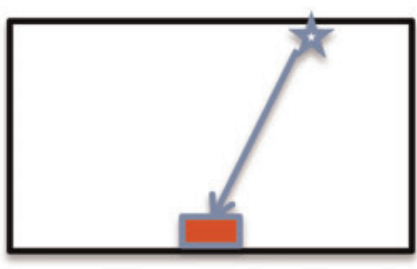

FLS trainer

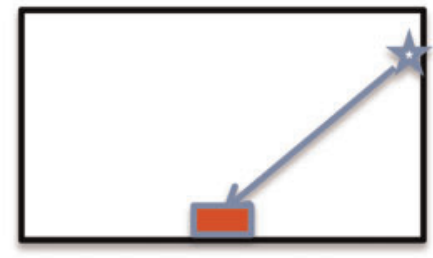

HM trainer

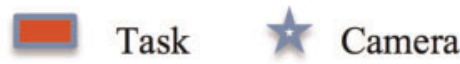

\section{FLS - Fundamentals of Laparoscopic Surgery \\ $\mathrm{HM}$ - Home-made}

Figure 3. Diagrammatic representation of the camera position in the FLS and home-made trainer.

people with no background in engineering and construction. The main advantages of our HM trainer are that it is minimalistic in its design, utilizes common materials, requires minimal engineering skills and yet retains the most fundamental features of a laparoscopic apparatus. As with all box trainers, the in vivo intra-abdominal experience is impossible to replicate with high fidelity in our opinion. However, given that the main aim of a box trainer is not to perform complex manoeuvres but to acquire basic laparoscopic skills, e.g. cutting, transferring and suturing, these limitations are acceptable.

Most home-made trainers in the literature have not been validated. Two studies ${ }^{13,14}$ validated their trainers by comparing the times to perform certain tasks against the commercially available trainers; hence we have adopted the same method of validating our trainer. In the validation component of this study, participants performed peg transfer significantly faster using the FLS trainer. One explanation is that the camera in the FLS trainer is fixed on the roof, giving a more acute angle, whereas the camera on HM trainer was fixed on the back wall, giving a less acute angle (Fig. 3). This allows easier performance or visualization of peg transfer in the FLS trainer, a task that requires more vertical movement than horizontal movement. Conversely, participants performed the pattern cut significantly faster using the HM trainer than the FLS trainer, likely for the same reason, because pattern cut requires more horizontal movement than vertical movement. These differences in time, in our opinion, are not clinically relevant.

The HM trainer was well received by most participants, and feedback was positive. Despite its low cost, the HM trainer's performance (lighting, video quality, simplicity of setup) is acceptable and so is its training capacity, in that it provides a realistic platform for training in laparoscopic skills comparable with the FLS trainer. Many trainees would consider making their own and using it. Having an HM trainer allows trainees to practise in their own time and space, and this could expedite their learning and mastery of basic laparoscopic skills. We would add that many inexpensive and creative laparoscopic tasks could be designed to aid skills training. For instance, a tissue handling skill can be developed by transferring matches from one plate to another or by peeling fibres off an orange; depth perception and hand-eye coordination can be improved by running thread through holes of different sizes placed at different levels and angles. ${ }^{10}$

This study had some limitations. First, the numbers used in the validation study are small. Second, despite our expectation of no statistically significant difference in results in the tasks between FLS and HM trainers, due to the aforementioned difference in design, certain tasks were easier on one or the other trainer. We feel that this should not affect laparoscopic training, because even in practice, many intra-operative factors are variable, e.g. assistants who hold the laparoscope, types of laparoscope, monitor and quality of image, etc. One must learn to deal with the limitation or challenge presented in every situation.

\section{Conclusion}

The HM laparoscopic trainer can be constructed cheaply and easily. It is comparable with the FLS trainer and is a viable alternative for trainees who wish to practise basic laparoscopic skills at home. 


\section{Conflicts of interest}

No conflict of interest declared.

\section{Acknowledgement}

The authors would like to acknowledge Dave Benjabutr for his contribution in data collection in this study. We would also like to thank Covidien for donating disposable laparoscopic graspers and endo-loops for this study.

\section{References}

1. Al-Abed Y, Cooper DG. A novel home laparoscopic simulator. J Surg Educ 2009; 66: 1-2. https://doi.org/10.1016/j.jsurg.2008. 08.002 .

2. Derossis A, Bothwell J, Sigman H, Fried GM. The effect of practice on performance in a laparoscopic simulator. Surg Endosc 1998; 12, 1117-1120. https://doi.org/10.1007/s00464 9900796.

3. Sturm LP, Windsor JA, Cosman PH, Cregan P, Hewett PJ, Maddern GJ. A systematic review of skills transfer after surgical simulation training. Ann Surg 2008; 248: 166-179. https://doi.org/10.1097/SLA.0b013e318176bf24.

4. Sroka G, Feldman LS, Vassiliou MC, Kaneva PA, Fayez R, Fried GM. Fundamentals of laparoscopic surgery simulator training to proficiency improves laparoscopic performance in the operating room-a randomized controlled trial. Am J Surg 2010; 199: 115-120. https://doi.org/10.1016/j.amjsurg.2009.07.035.

5. Hafford ML, Van Sickle KR, Willis RE, Wilson TD, Gugliuzza K, Brown KM, et al. Ensuring competency: are fundamentals of laparoscopic surgery training and certification necessary for practicing surgeons and operating room personnel? Surg Endosc 2013; 27: 118-126. https://doi.org/10.1007/s00464-012-2437-7.
6. Marlow N, Altree M, Babidge W, Field J, Hewett P, Maddern GJ. Basic laparoscopic skills acquisition. ANZ J Surg 2014; 84: 976-980. https://doi.org/10.1111/ans.12282.

7. Satava RM. Historical review of surgical simulation-a personal perspective. World J Surg 2008; 32: 141-148. https://doi. org/10.1007/s00268-007-9374-y.

8. Limb \& Things Ltd. Available from: https://limbsandthings. com/global/products/50301/50301-limbs-things-laparoscopicskills-trainer-usb.

9. 3-Dmed. Available from: https://www.3-dmed.com/productcategory/mits-series/t3-series/.

10. Walczak DA, Piotrowski P, Jędrzejczyk A, Pawełczak D, Pasieka Z. A laparoscopic simulator-maybe it is worth making it yourself. Wideochir Inne Tech Maloinwazyjne 2014; 9: 380-386. https://doi.org/10.5114/wiitm.2014.44139.

11. Pokorny MR, McLaren SL. Inexpensive home-made laparoscopic trainer and camera. ANZ J Surg 2004; 74: 691-693. https://doi.org/10.1111/j.1445-1433.2004.03121.x.

12. Gue S. Home-made videoscopic trainer for operative laparoscopic surgery. Aust N Z J Surg 1995; 65: 820-821. https://doi. org/10.1111/j.1445-2197.1995.tb00568.x.

13. Wong J, Bhattacharya G, Vance SJ, Bistolarides P, Merchant AM. Construction and validation of a low-cost laparoscopic simulator for surgical education. J Surg Educ 2013; 70: 443450. https://doi.org/10.1016/j.jsurg.2013.02.004.

14. Yoon R, Del Junco M, Kaplan A, Okhunov Z, Bucur P, Hofmann M, et al. Development of a novel iPad-based laparoscopic trainer and comparison with a standard laparoscopic trainer for basic laparoscopic skills testing. J Surg Educ 2015; 72: 41-46. https://doi.org/10.1016/j.jsurg.2014.06.011.

15. Chen X, Pan J, Chen J, Huang H, Wang J, Zou L, et al. A novel portable foldable laparoscopic trainer for surgical education. J Surg Educ 2016; 73: 185-189. https://doi.org/10. 1016/j.jsurg.2015.11.004. 